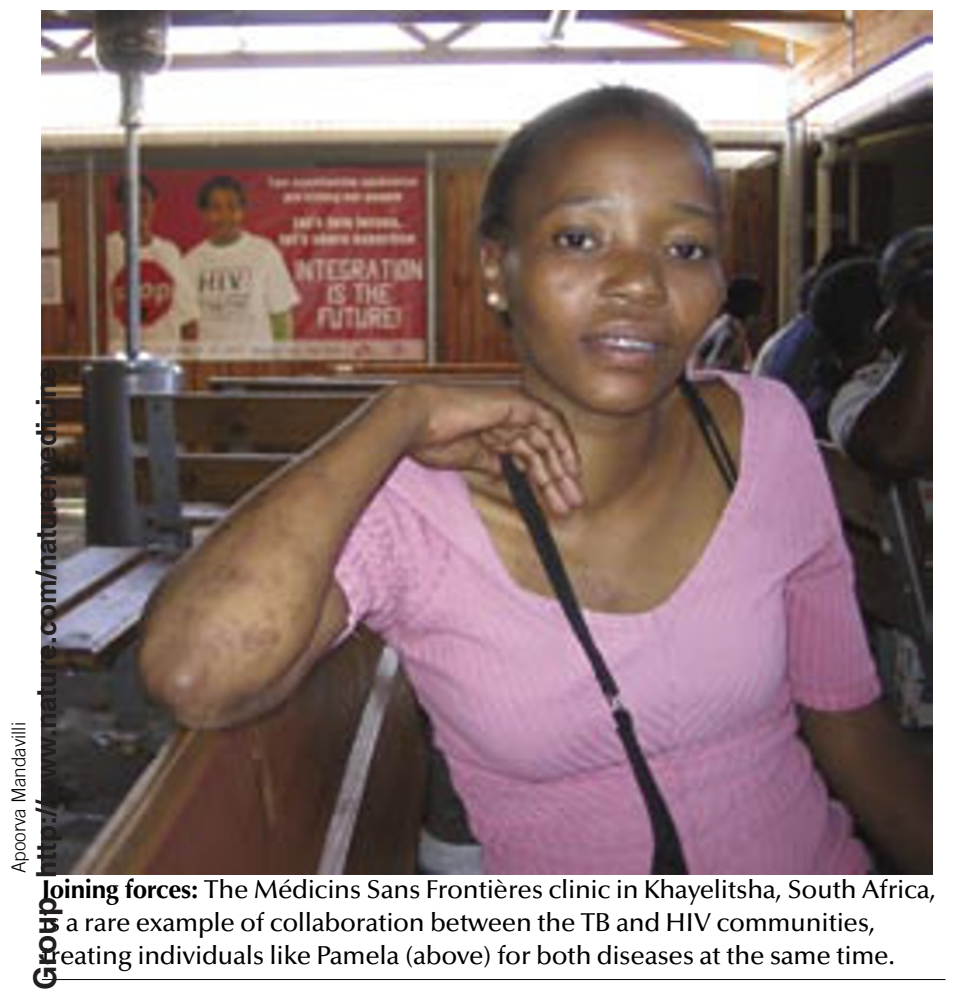

case definition to therapeutic guidelines to standard operating procedures, it's all written in stone and cast," says Goemaere. "I've never seen so many guidelines. It's almost Stalinian.”

The HIV community, in contrast, driven in part by activists, is focused on empowering the individual, with a heavy emphasis on applying the latest research.

The HIV-TB crisis has resulted in a power struggle between these two cultures, leading up all the way to the WHO's own TB and HIV programs. "Everyone wonders who's going to swallow

O even though those results often inform policy the other," says Goemaere. decisions.

A decade ago, "the public health community was accused of being philistine or narrowminded at best and alienated from the basic science community," says Christopher Dye Nunn's colleague at the Stop TB program.

"There was some sense that we were enemies of each other and all this kind of nonsensenot anymore," Dye says. "It's way better than it was about ten years ago."

Maybe so. But Nunn's perspective is still far removed from the urgency with which most researchers on the ground speak of IRIS.

"The public health people who ignore the clinical importance of things, they undermine their credibility in the eyes of the people who run the program," says Chaisson. "You need a public health approach, but you need to take care of patients."

\section{Power struggle}

This disconnect is even more stark in the radically different cultures of the HIV and TB communities.

$\mathrm{TB}$ is an old disease and its approach, set primarily by the WHO, is faithful to the principles of classic public health: doing the best for the most people. With the perception that TB was curable, interest in TB research also dried up, and things are done much the same way they were decades ago.

"It's extremely rigid from
But there are small signs that things are changing, beginning with the WHO's influence on research for AIDS and tuberculosis (TB). have resource in the community. more funds. raise the awareness of the governments," he says.

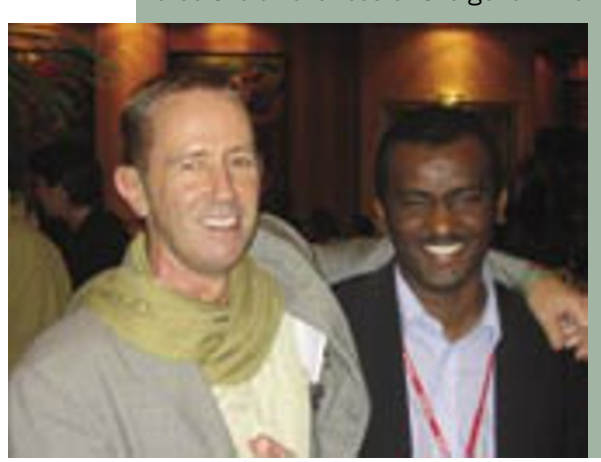

Mark Harrington (left) with the WHO's Haileyesus

Getahun. admission that DOTS, the cornerstone of its TB programs, must take HIV into account (see box, page 269).

The WHO now recommends HIV testing for those infected with TB, preventive TB drugs for HIV-positive people and integration of TB and HIV programs.

In Rwanda and Kenya, $75 \%$ of TB sufferers are being tested for HIV, notes Nunn. "That's up from nothing about a year and a half ago, that's progress on an enormous scale," he says. "We have illustrated that it can be done." The WHO is meeting in March to expand the pilot scheme to more countries.

In South Africa, too, the integrated clinic in Khayelitsha is a resounding success, overflowing with those who are happy to get all their medicines in one place. The integrated programs may also help answer some fundamental questions about $\mathrm{TB}$ pathogenesis.

Researchers from the University of Cape Town are collecting samples from those who come to the clinics, studying HIV's effect on TB progression and perhaps, by extension, what protects people from TB, says Wilkinson. "This is one of the greatest experiments in public health ever invented."

Apoorva Mandavilli is Nature Medicine's senior news editor.

\title{
Spotlight on... Mark Harrington
}

For a liberal arts graduate with no formal science background, Mark Harrington has a lot of

The head of the New York-based activist organization Treatment Action Group (TAG), Harrington commands respect from even the most senior AIDS and TB researchers. Last year, the group published a comprehensive analysis of funding for TB - showing, for example, that TB gets less than five percent of the amount spent on AIDS - that has quickly become a must-

Harrington himself sits on the advisory boards of powerful agencies, including the US National Institutes of Health and the World Health Organization. With a four-year, $\$ 4.7$ million grant from the Bill \& Melinda Gates Foundation, he is trying to strengthen support for TB by training AIDS activists on the issues, coordinating outreach with African activist groups, educating advocates and policy makers in Washington, DC, and pushing for

"We need to have strong activists in the countries most affected by HIV and TB so they can

Harrington is no stranger to advocacy. In 1988 , during the early days of the AIDS movement, he joined the famous ACT UP group in New York. He launched TAG four years later. Though still heavily involved in AIDS activism, Harrington in 2002 began campaigning for TB, a neglected disease with far fewer advocates.

"TB is a disease of the poor and the excluded and there's never going to be a worldwide movement of TB activists the way there is with HIV," says Harrington. "We're not going to get to where we need to be without a massive investment into basic science."

Alisa Opar, New York 